\title{
Microstructural brain injury in post-concussion syndrome after minor head injury
}

\author{
Marion Smits • Gavin C. Houston • Diederik W. J. Dippel • Piotr A. Wielopolski • \\ Meike W. Vernooij • Peter J. Koudstaal • M. G. Myriam Hunink • Aad van der Lugt
}

Received: 29 July 2010 / Accepted: 17 September 2010 / Published online: 6 October 2010

(C) The Author(s) 2010. This article is published with open access at Springerlink.com

\begin{abstract}
Introduction After minor head injury (MHI), postconcussive symptoms commonly occur. The purpose of this study was to correlate the severity of post-concussive symptoms in MHI patients with MRI measures of microstructural brain injury, namely mean diffusivity (MD) and fractional anisotropy (FA), as well as the presence of microhaemorrhages.

Methods Twenty MHI patients and 12 healthy controls were scanned at $3 \mathrm{~T}$ using diffusion tensor imaging (DTI) and high-resolution gradient recalled echo (HRGRE) T2*weighted sequences. One patient was excluded from the analysis because of bilateral subdural haematomas. DTI data were preprocessed using Tract Based Spatial Statistics.
\end{abstract}

M. Smits $(\square) \cdot$ P. A. Wielopolski $\cdot$ M. W. Vernooij

M. G. M. Hunink · A. van der Lugt

Department of Radiology (Hs-224),

Erasmus MC-University Medical Centre Rotterdam,

PO Box 2040, 3000 CA Rotterdam, the Netherlands

e-mail: marion.smits@erasmusmc.nl

G. C. Houston

Applied Science Lab, GE Healthcare,

's Hertogenbosch, the Netherlands

D. W. J. Dippel · P. J. Koudstaal

Department of Neurology,

Erasmus MC-University Medical Centre Rotterdam,

Rotterdam, the Netherlands

M. G. M. Hunink

Department of Epidemiology,

Erasmus MC-University Medical Centre Rotterdam,

Rotterdam, the Netherlands

M. G. M. Hunink

Department of Health Policy \& Management,

Harvard School of Public Health,

Boston, MA, USA
The resulting MD and FA images were correlated with the severity of post-concussive symptoms evaluated with the Rivermead Postconcussion Symptoms Questionnaire. The number and location of microhaemorrhages were assessed on the HRGRE T2*-weighted images.

Results Comparing patients with controls, there were no differences in MD. FA was decreased in the right temporal subcortical white matter. MD was increased in association with the severity of post-concussive symptoms in the inferior fronto-occipital fasciculus (IFO), the inferior longitudinal fasciculus and the superior longitudinal fasciculus. FA was reduced in association with the severity of post-concussive symptoms in the uncinate fasciculus, the IFO, the internal capsule and the corpus callosum, as well as in the parietal and frontal subcortical white matter. Microhaemorrhages were observed in one patient only.

Conclusions The severity of post-concussive symptoms after MHI was significantly correlated with a reduction of white matter integrity, providing evidence of microstructural brain injury as a neuropathological substrate of the postconcussion syndrome.

Keywords Craniocerebral trauma $\cdot$ Post-concussion syndrome $\cdot$ Diffusion tensor imaging $\cdot$ Magnetic resonance imaging $\cdot$ Cognition disorder

\section{Introduction}

Head injury is one of the most common injuries in the Western world, with minor head injury accounting for 70 $90 \%$ of the head injury cases [1]. Despite being classified as minor, as clinically determined by a normal or near-normal level of consciousness (Glasgow Coma Scale (GCS) score of 13-15) and a brief period of loss of consciousness or 
posttraumatic amnesia, a large proportion of patients suffer from a wide variety of symptoms for months after the injury [2-5]. This so-called post-concussion syndrome includes symptoms such as headache, fatigue and cognitive complaints such as memory and attention deficits. Despite the subjective severity of these symptoms, conventional imaging with computed tomography (CT) or magnetic resonance (MR) imaging is generally normal. Upon neuropsychological examination, cognitive deficits, if any, are subtle and most often in the executive domain [6-8]. Symptoms persisting for more than 3 to 6 months are difficult to treat and can lead to vocational disability $[9,10]$, representing a substantial burden to society and healthcare services. The apparent discrepancy between the subjective severity of complaints and the relative lack of objective neuropsychological and imaging findings has led to controversy about this syndrome, and the exact aetiology as well as the neuropathological substrate remain unclear. Age, gender, previous disease, substance abuse, litigation and emotional factors have all been suggested to be associated with the occurrence and persistence of symptoms, but correlations are inconsistent $[2,11]$.

A widely accepted hypothesis for an organic origin of the post-concussion syndrome postulates that the symptoms are due to microstructural white matter damage as a result of straining, stretching, deforming or even shearing forces, which is not detectable with conventional neuroimaging [12-16]. This hypothesis is indirectly supported by findings from single photon emission computed tomography and perfusion $\mathrm{CT}$, showing hypoperfusion in the frontal and parietal lobes of minor head injury patients in general $[9,17]$, and magnetisation transfer imaging, showing a global reduction of the magnetic transfer ratio in minor head injury patients with post-concussive symptoms [9, 18]. Recent work in minor head injury patients shows disruption of saccadic eye movements, indicative of damage to midbrain pathways [19], and differences in event-related brain potentials, indicating disrupted speed of processing and connectivity, implicating white matter dysfunction [20]. Further support is provided by a positive correlation between post-concussive symptoms and serum concentrations of protein S100-b, which is found in high concentrations in glial cells and Schwann cells and is highly specific for lesions of the central nervous system [21,22].

The traumatic axonal injury, which includes both diffuse axonal injury as well as less severe degrees of axonal damage, presumed to underlie the microstructural injury, typically occurs during rapid acceleration and/or deceleration trauma mechanisms at interfaces of tissues with differences in density and rigidity, such as at the corticomedullary junction (subcortical white matter), as well as in the corpus callosum and the rostral brainstem adjacent to the cerebellar peduncles (pontine-mesencephalic junction) [12, 23-27].
Two advanced MR neuroimaging techniques, diffusion tensor imaging (DTI) and 3D high-resolution (submillimetre) gradient recalled echo (HRGRE) T2*-weighted imaging $[28,29]$, are used to detect axonal injury in vivo with higher sensitivity [30-34] and to provide direct evidence of microstructural brain damage after minor head injury. Diffusion-weighted imaging (DWI) provides image contrast sensitive to differences in the diffusion of water molecules [35-37]. Thus, DWI offers an in vivo assessment of cell integrity and pathology of the white matter by deriving the mean diffusivity as a measure of the magnitude of diffusion. With DTI, in which diffusion-weighted gradients are applied in multiple directions, the anisotropy of diffusion can be assessed in addition to mean diffusivity. A higher anisotropy of diffusion reflects a motion of water molecules favoured in a specific direction, such as parallel to highly structured white matter fibres. A reduction of anisotropy is generally thought to reflect a reduction of the integrity of white matter fibres, such as in traumatic axonal injury, including diffuse axonal injury [25, 30-32, 35, 38]. Axonal injury lesions resulting from true shearing injury may be haemorrhagic [25]. Improved detection of these microhaemorrhagic shearing lesions is attained with higher field strength [27], as well as HRGRE T2*-weighted sequences as compared with conventional gradient-echo sequences [39-41].

The purpose of this study was to identify the neuropathological correlate of post-concussive symptoms using advanced MRI techniques. Specifically, we correlated the severity of post-concussive symptoms with focal changes in diffusivity as well as the presence of microhaemorrhages in minor head injury patients in whom $\mathrm{CT}$ and conventional MR imaging did not show traumatic abnormalities.

\section{Methods}

\section{Study population}

Patients were prospectively and consecutively included 1 month after presentation to our emergency department with blunt head trauma if they met the following inclusion criteria: aged 18-50 years, a GCS score of $13-15$ and a normal neurological examination upon presentation, as well as a normal $\mathrm{CT}$ of the head performed within $24 \mathrm{~h}$ of injury. As a control group, healthy volunteers matched for age, gender and educational level were recruited from the included patients' peers and family, where possible, and additionally from hospital co-workers.

Head injury patients and controls were excluded if they had a history of neurological or psychiatric disease, had previous head injury, used prescription medication other than oral contraceptives or had contraindications for MR imaging. 
The study protocol was approved by the institution's ethical review board and has therefore been performed in accordance with the ethical standards laid down in the 1964 Declaration of Helsinki, and written informed consent was obtained from all participants.

\section{Participant characteristics}

General demographic data were collected from all participants. Educational level was classified as follows: (1) primary education only; (2) lower-level secondary education; (3) middle-level secondary education; and (4) higher-level secondary or post-secondary education. All participants underwent general neurological examination and testing of crude cognitive function with the Mini Mental Status Examination (MMSE) [42].

In the head injury patients, the number and severity of postconcussive complaints was assessed by means of the Rivermead Postconcussion Symptoms Questionnaire (RPSQ) [43]. The RPSQ is a five-point-scale of 16 symptoms that are common after head injury and has a high test-retest and interrater agreement for the assessment of both the presence and the severity of post-concussive symptoms [44, 45]. Patients rate severity of each symptom in comparison with pre-injury levels on a scale from 0 (no or not more symptoms than before the injury) to 4 (much more severe symptoms than before the injury), thus adjusting for the high base rate of (some of these) symptoms in the general population. Additional symptoms may be recorded and rated similarly. The higher the sum score, the more (severely) symptoms are present after the injury. Additionally, the number of days after which the patients had returned to work or school was recorded.

\section{MRI acquisition protocol}

Imaging was performed on a 3-T MR system (HD platform, GE Healthcare, Milwaukee, WI, USA). An eight-channel head coil was used for reception of the signal. For anatomical reference, a high-resolution 3D fast spoiled gradient-echo T1-weighted image with an inversion recovery preparation pulse was acquired, with the following pulse sequence parameters: repetition time (TR)/echo time (TE)/ inversion time (TI) $10.7 / 2.2 / 300 \mathrm{~ms}$, flip angle $18^{\circ}$, acquisition matrix $416 \times 256$, field of view (FOV) $250 \times 175 \mathrm{~mm}^{2}$, 192 slices with a slice thickness of $1.6 \mathrm{~mm}$ and $0.8-\mathrm{mm}$ overlap and acquisition time of 4 min and $57 \mathrm{~s}$.

As a highly sensitive sequence for the detection of white matter brain injury [46], a fluid-attenuated inversion recovery (FLAIR) acquisition was obtained with the following pulse sequence parameters: TR/TE/TI 8,000/120/2,000 ms, acquisition matrix $256 \times 128$, FOV $210 \times 210 \mathrm{~mm}^{2}, 64$ contiguous slices with a slice thickness of $2.5 \mathrm{~mm}$ and acquisition time of $3 \mathrm{~min}$ and $13 \mathrm{~s}$.
For DTI, we used a 2D single-shot, spin-echo diffusionweighted EPI acquisition with TR/TE 14,200/68.9 ms, acquisition matrix $64 \times 128$, FOV $220 \times 220 \mathrm{~mm}^{2}, 70$ contiguous slices with a slice thickness of $2.0 \mathrm{~mm}$, array spatial sensitivity encoding technique (ASSET) acceleration factor 2 and acquisition time of $6 \mathrm{~min}$ and $38 \mathrm{~s}$. One image with a $b$ value of $0 \mathrm{~s} / \mathrm{mm}^{2}$ was acquired, and for diffusionweighted images, a $b$ value of $1,000 \mathrm{~s} / \mathrm{mm}^{2}$ was used, acquired in 25 non-collinear directions.

High-resolution $\mathrm{T} 2 *$-weighted images were acquired with a 3D low-bandwidth, HRGRE acquisition with the following pulse sequence parameters: TR/TE $43 / 29.5 \mathrm{~ms}$, flip angle $14^{\circ}$, bandwidth $88 \mathrm{~Hz} /$ pixel, acquisition matrix $512 \times 320$, FOV $260 \times 156 \mathrm{~mm}^{2}, 128$ contiguous slices with slice thickness of $1.0 \mathrm{~mm}$, ASSET acceleration factor 2 and acquisition time of $9 \mathrm{~min}$ and $14 \mathrm{~s}$.

Data analysis

\section{Participant characteristics}

We tested differences in participant characteristics between patients and controls for significance $(p<0.05)$ with the Student's $t$ test for continuous (age, MMSE), Pearson's chisquare test for categorical (gender) and Kruskal-Wallis for ordinal (educational level) variables using the Statistical Package for Social Sciences version 15.0 (SPSS Inc., Chicago, IL, USA).

\section{Conventional structural imaging}

The T1-weighted and FLAIR images were transferred to a workstation and reviewed by two radiologists (A.L. and M.S.) in consensus for abnormalities consistent with traumatic brain injury. Participants with traumatic brain injury abnormalities on either of these two sequences were excluded from the analysis.

\section{DTI analysis}

The DTI data were transferred to a workstation and spatially pre-processed using Tract Based Spatial Statistics (TBSS) [47], part of the FMRIB Software Library (FSL, Analysis Group, FMRIB, Oxford, UK) [48].

First, DTI data were corrected for head motion and eddy current artefacts. Mean diffusivity (MD) and fractional anisotropy (FA) maps were created by fitting a tensor model to the raw diffusion data using FDT and then brain-extracted using BET based on the $b=0$ image [49]. All participants' FA data were then aligned into $1 \times 1 \times 1 \mathrm{~mm}^{3}$ Montreal Neurological Institute (MNI) common space using the nonlinear registration image registration toolkit $[50,51]$. The mean FA image was created and thinned to create a mean FA skeleton 
representing the centres of all tracts common to the group, using an FA-threshold of 0.20. Each participant's aligned MD and FA maps were then projected onto this skeleton.

Voxel-wise statistical analysis of the skeletonised white matter voxels was performed with Statistical Parametric Mapping version 8 (SPM8, Wellcome Department University College London, London, UK) implemented in Matlab version 7.5.0 (The Mathworks, Sherborn, MA, USA). Using two-sample $t$ tests, patients' and controls' MD and FA maps were tested for differences. Additionally, in a univariable linear regression analysis, we correlated the patients' individual MD and FA images with their RPSQ scores. Results were thresholded on a cluster level at $p<0.05$ (with false discovery rate correction for multiple comparisons) and size $\geq 5$ voxels.

The thresholded statistical T-contrast maps were projected on the mean FA images. For display purposes, the thresholded contrast maps of single voxel thickness were thickened to fill the entire local tracts as seen on the mean FA image. Anatomical labelling was performed manually using the DTI atlases provided by Hermoye et al. [52] and Wakana et al. [53]. A distinction was made between the centrally located white matter tracts (fasciculi, corpus callosum) and the more peripherally located subcortical white matter, consisting of fibres originating from the major white matter tracts.

\section{Microhaemorrhage assessment}

The 3D HRGRE T2*-weighted images were transferred to a workstation. The number and location of microhaemorrhages were recorded by two experts in reading 3D HRGRE T2*-weighted images (M.V. and A.L.) [40, 41] who were unaware of the subjects' RPSQ scores.

\section{Results}

Study population

Between December 2005 and November 2006, 236 patients with recent minor head injury presented to our emergency department who were eligible for inclusion in the study. Of these, 51 could be contacted, 36 of whom were willing to participate. Of these, six did not fulfil the study's inclusion criteria and nine were excluded because of contraindications for MR imaging $(n=2)$, previous history of neurological or psychiatric disease $(n=4)$ and previous history of head injury $(n=3)$. One patient did not complete the entire scanning session due to claustrophobia. The 20 remaining patients were imaged at an average of 30.6 days (range, 1840 days) after the injury. In 19 patients, no traumatic abnormalities were observed on the T1-weighted and FLAIR images. One patient was found to have bilateral subdural haematomas and was excluded from the analysis. Additionally, 12 healthy volunteers were imaged.

\section{Participant characteristics}

The majority of participants were male $(n=18,58 \%)$ and the mean age was 26.4 years (range, 18-50 years). Neurological examination was normal in all participants. There was no difference between patients and controls regarding age $(p=0.47)$, gender $(p=0.49)$, educational level $(p=0.42)$ or crude cognitive function as measured with the MMSE ( $p=0.91$; Table 1$)$. The mean patient RPSQ score was 15 (median, 5; range, $0-46$ ). On average, patients were able to return to work 10 days after the injury, whilst two patients had not returned to work at the time of the study.

All but one patient had a history of loss of consciousness or (posttraumatic) amnesia after the injury. Most patients had a GCS score of 15 upon presentation $(n=13,68.4 \%)$; six patients presented with a GCS score of 14 .

\section{DTI analysis}

When comparing patients with controls, no differences were found in MD. One area of decreased FA was found in patients compared with controls in the right temporal lobe subcortical fibres of the inferior fronto-occipital fasciculus (IFO; MNI coordinates, 51-61 1; cluster size, 13 voxels; $T$ value, 5.65; Fig. 1).

When correlating the patients' MD maps with their RPSQ scores, a significant increase of MD associated with the severity of post-concussive symptoms was seen in the left IFO and inferior longitudinal fasciculus (ILF), as well as in the superior longitudinal fasciculus (SLF; Table 2 and Fig. 2). No decrease in MD was seen.

A significant reduction of FA in association with the severity of post-concussive symptoms was seen in the right uncinate and IFO, the posterior limb of the internal capsule bilaterally, the splenium of the corpus callosum on the right side, as well as in peripheral white matter consisting of fibres originating from the corpus callosum in the left parietal and right frontal lobes (Table 3 and Fig. 3).

Table 1 Participant characteristics

\begin{tabular}{lcll}
\hline & $\begin{array}{l}\text { Patients } \\
(n=19)\end{array}$ & $\begin{array}{l}\text { Controls } \\
(n=12)\end{array}$ & $p$ value \\
\hline Age, years (SD) & $26(7.4)$ & $28(10)$ & 0.47 \\
Male gender, $n(\%)$ & $10(53)$ & $8(67)$ & 0.49 \\
Educational level, mean (SD) & $3.2(1.0)$ & $3.3(1.1)$ & 0.42 \\
MMSE, mean (SD) & $27(1.8)$ & $28(1.8)$ & 0.91 \\
GCS score, mean (SD) & $15(0.5)$ & - & - \\
RPSQ score, mean (SD) & $15(16)$ & - & - \\
\hline
\end{tabular}


Fig. 1 Mean FA images in axial, coronal and sagittal view showing significantly reduced FA in patients compared with controls in the right temporal lobe subcortical fibres of the inferior fronto-occipital fasciculus

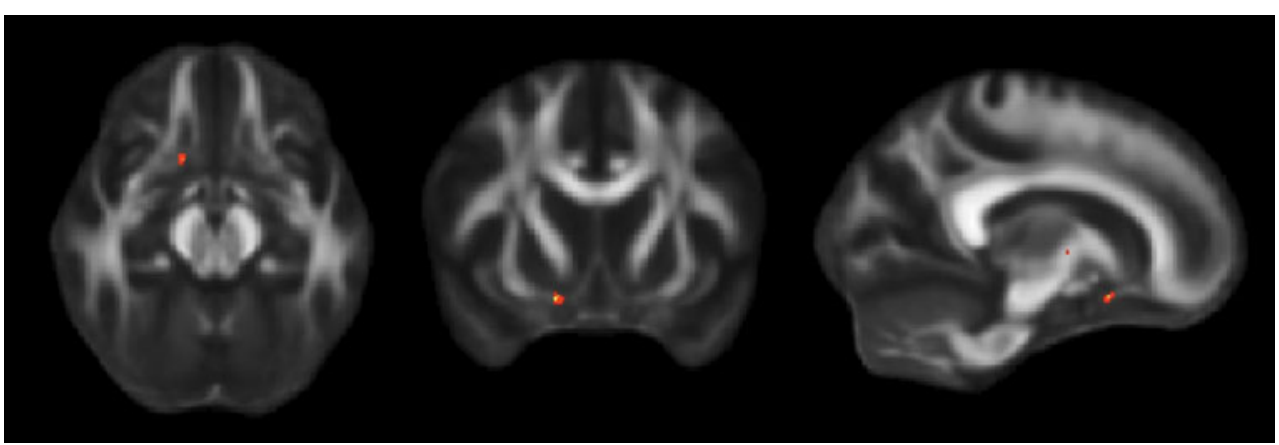

There was no significant increase of FA in relation to the severity of post-concussive symptoms.

Microhaemorrhage assessment

Microhaemorrhages were observed in one patient only (female, 18.5 years of age), localised in the right frontal lobe (Fig. 4). Although this patient had severe postconcussive symptoms (RPSQ score=21), the scarcity of these lesions does not allow any inferences on their association with post-concussive symptoms.

\section{Discussion}

In this study of minor head injury patients, we found a significant relationship between the severity of postconcussive symptoms of minor head injury and microstructural white matter changes in the absence of macrostructural traumatic abnormalities.

Post-concussive symptoms are common after minor head injury [2-5], but their organic origin has been debated due to the poor correlation between objective imaging abnormalities and the degree of symptoms. Consistently, both autopsy and in vivo longitudinal volumetric studies report evidence of much more widespread and generalised damage to the brain than visualised with single time point conventional imaging, which has led to the generally accepted idea that conventional neuroimaging studies with CT and MRI underestimate the true extent of brain damage

Table 2 Areas of significant positive correlation of MD and RPSQ score in patients only

\begin{tabular}{|c|c|c|c|c|c|c|}
\hline \multirow{2}{*}{$\begin{array}{l}\text { Anatomical } \\
\text { location }\end{array}$} & \multirow[t]{2}{*}{ Side } & \multicolumn{3}{|c|}{ MNI coordinates } & \multirow{2}{*}{$\begin{array}{l}\text { Cluster size } \\
\text { (no. of voxels) }\end{array}$} & \multirow[t]{2}{*}{$T$ value } \\
\hline & & $x$ & $y$ & $z$ & & \\
\hline SLF (parietal lobe) & $\mathrm{L}$ & -35 & -30 & 27 & 11 & 6.24 \\
\hline $\begin{array}{l}\text { IFO/ILF } \\
\text { (occipital lobe) }\end{array}$ & $\mathrm{L}$ & -32 & -79 & -2 & 8 & 5.35 \\
\hline
\end{tabular}

after head injury [12]. The hypothesis that post-concussive symptoms are the result of microstructural brain injury is therefore well recognised.

The microstructural injury is thought to consist of traumatic axonal injury, ranging from the more severe diffuse axonal or true shearing injury to milder degrees of axonal damage. Rotational acceleration and/or deceleration forces occurring at the time of the injury are thought to induce maximal damage, with the corpus callosum, rostral brainstem and subcortical white matter as predilection sites. Empirical evidence of damage in these regions has been observed in postmortem brains even following very brief periods of recorded loss of consciousness [12]. Three stages of traumatic axonal injury are recognised. The first consists of a biochemical alteration in which the minimally stretched axons do not tear and changes may be transient. In the second stage, the cytoskeleton itself is damaged, accompanied by local swelling and enlargement of the injured axon. In the third stage, axotomy occurs, either primarily or secondarily, in which the axon is severed and antero- and retrograde degeneration follows, leading to a disproportionate reduction in white matter, evidenced by the wellrecognised global white matter atrophy in the later stages after head injury.

FLAIR and GRE T2*-weighted sequences are the two conventional MR imaging sequences that are most sensitive to the secondary white matter changes, which may occur in any traumatic axonal injury, and haemorrhage, occuring in the more severe diffuse axonal or true shearing injury, respectively $[30,46]$. The histological changes in traumatic axonal injury, such as changes in axolemmal permeability and misalignments of the cytoskeletal network, however, are not detectable with conventional structural imaging techniques. DTI offers the unique possibility of detecting these histological effects in vivo by measuring alterations of mean diffusivity and diffusion anisotropy. Mouse models have demonstrated a good correlation between traumatic axonal injury lesions and reduction of white matter anisotropy measured with DTI [54]. The interpretation of results from such studies in humans is confounded by the substantial heterogeneity of the patient populations studied 
Fig. 2 Mean fractional anisotropy images in axial, coronal and sagittal view showing significantly increased mean diffusivity in association with the severity of post-concussive symptoms (patient group regression analysis) in the left superior longitudinal fasciculus (a) and inferior frontooccipital/inferior longitudinal fasciculus (b)
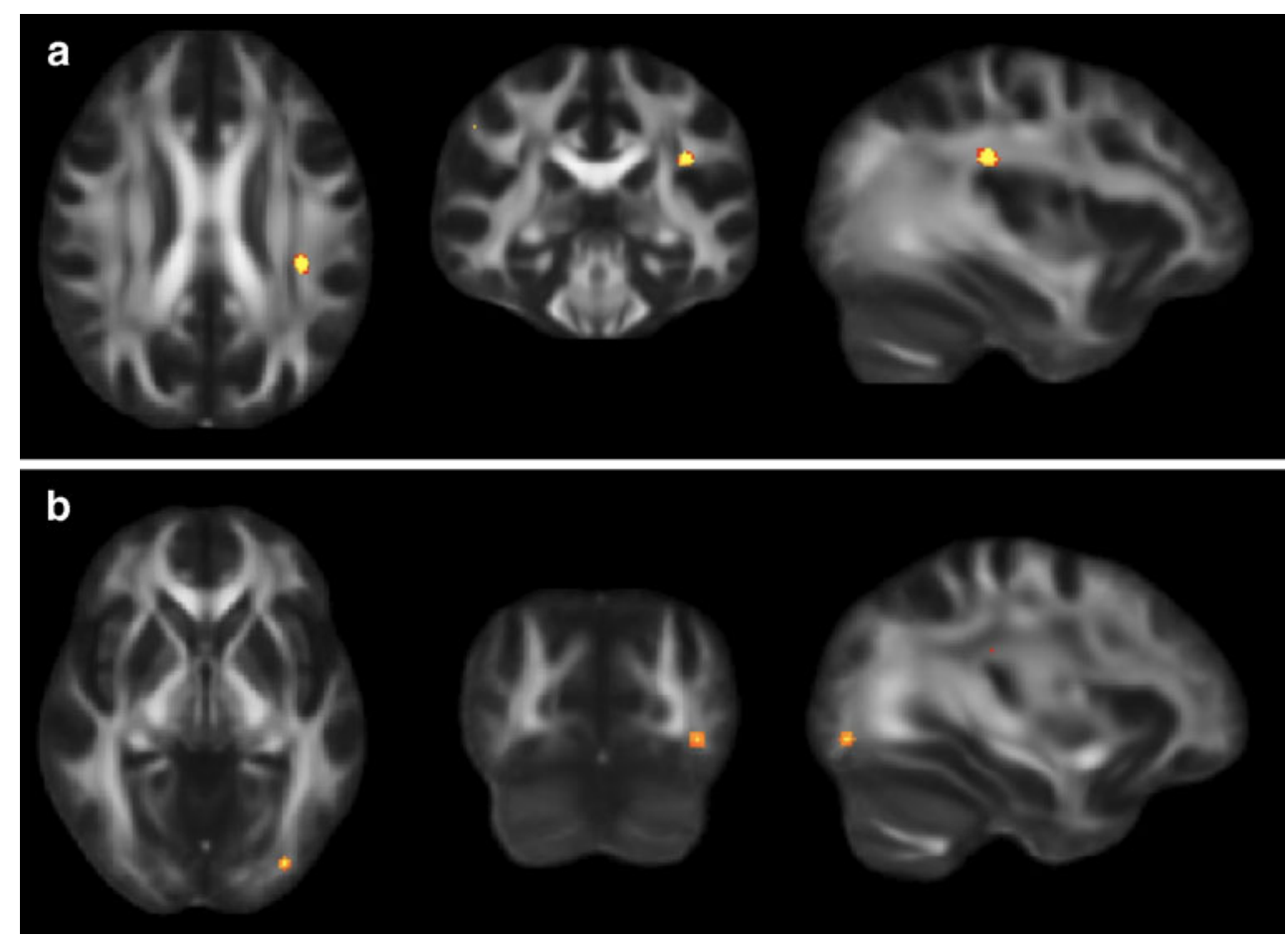

in which patients with all degrees of head injury, irrespective of the presence or severity of post-concussive symptoms, are compared with healthy controls. Nonetheless, reductions of anisotropy have been reported in typical locations of the more severe diffuse axonal injury, such as the corpus callosum [23, 26, 30, 31, 55-57] and the mesencephalon [32], as well as in

Table 3 Areas of significant negative correlation between FA and RPSQ in patients only

\begin{tabular}{|c|c|c|c|c|c|c|}
\hline \multirow{2}{*}{$\begin{array}{l}\text { Anatomical } \\
\text { location }\end{array}$} & \multirow[t]{2}{*}{ Side } & \multicolumn{3}{|c|}{ MNI coordinates } & \multirow{2}{*}{$\begin{array}{l}\text { Cluster size } \\
\text { (no. of voxels) }\end{array}$} & \multirow[t]{2}{*}{$T$ value } \\
\hline & & $x$ & $y$ & $z$ & & \\
\hline $\begin{array}{l}\text { Uncinate/IFO } \\
\text { (temporal lobe) }\end{array}$ & $\mathrm{R}$ & 35 & 2 & -15 & 8 & 4.49 \\
\hline $\begin{array}{l}\text { Uncinate/IFO } \\
\text { (temporal lobe) }\end{array}$ & $\mathrm{R}$ & 35 & -9 & -6 & 6 & 4.67 \\
\hline $\begin{array}{l}\text { Internal capsule } \\
\text { (posterior limb) }\end{array}$ & $\mathrm{L}$ & -8 & -1 & -5 & 5 & 5.92 \\
\hline $\begin{array}{l}\text { Internal capsule } \\
\text { (posterior limb) }\end{array}$ & $\mathrm{L}$ & -18 & -9 & 4 & 5 & 4.16 \\
\hline $\begin{array}{l}\text { Internal capsule } \\
\text { (posterior limb) }\end{array}$ & $\mathrm{R}$ & 17 & -3 & 6 & 6 & 4.99 \\
\hline $\begin{array}{l}\text { Corpus callosum } \\
\text { (splenium) }\end{array}$ & $\mathrm{R}$ & 17 & -43 & 26 & 7 & 4.17 \\
\hline $\begin{array}{l}\text { Callosal fibres } \\
\text { (parietal lobe) }\end{array}$ & $\mathrm{L}$ & -21 & -81 & 34 & 6 & 4.65 \\
\hline $\begin{array}{l}\text { Callosal fibres } \\
\text { (parietal lobe) }\end{array}$ & $\mathrm{L}$ & -32 & -68 & 21 & 9 & 4.19 \\
\hline $\begin{array}{l}\text { Callosal fibres } \\
\text { (frontal lobe) }\end{array}$ & $\mathrm{R}$ & 11 & 56 & -10 & 5 & 4.78 \\
\hline $\begin{array}{l}\text { Callosal fibres } \\
\text { (frontal lobe) }\end{array}$ & $\mathrm{R}$ & 17 & 52 & 13 & 6 & 4.70 \\
\hline
\end{tabular}

the internal capsule $[23,26,31-33,55-57]$, the uncinate fasciculus [56], the cerebral peduncle [32], the corticospinal tract [34], the medial temporal lobe [32], the IFO [56] and SLF $[32,34]$, the anterior and posterior cingulate $[33,56]$, and the anterior and posterior periventricular white matter [33]. We found areas of reduced anisotropy associated with the severity of post-concussive symptoms in the splenium of the corpus callosum, the internal capsule, and the uncinate fasciculus and IFO, as well as in the parietal and frontal subcortical white matter fibres. These foci of loss of white matter integrity closely resemble those previously reported in diffuse axonal injury.

When directly comparing patients with controls, however, we did not find any consistent differences in MD or FA. Similar findings are reported by Miles et al. [67] and Messé et al. [59] who also found white matter changes correlated with cognitive dysfunction and severity of post-concussive symptoms after minor head injury, respectively, in the absence of differences in white matter integrity between patients and controls. These findings may be explained by the fact that patients display a wide range of symptoms and symptom severity, with several patients having no symptoms at all and others having very severe symptoms. Such heterogeneity may obscure subtle differences between patients and controls.

The fact that the areas of significantly reduced anisotropy we found are correlated with the severity of postconcussive symptoms provides direct evidence that traumatic axonal injury is a significant contributing factor to symptom severity. Given the fact that only one of our 
Fig. 3 Mean FA images in axial, coronal and sagittal view showing areas of significantly reduced FA in association with the severity of post-concussive symptoms (patient group regression analysis) in the peripheral white matter consisting of fibres originating from the corpus callosum in the right frontal lobe (a), the right uncinate and inferior fronto-occipital fasciculus and left internal capsule (b), the posterior limb of the internal capsule bilaterally (c), the corpus callosum (d), and the peripheral white matter consisting of fibres originating from the corpus callosum in the left parietal lobe (e)
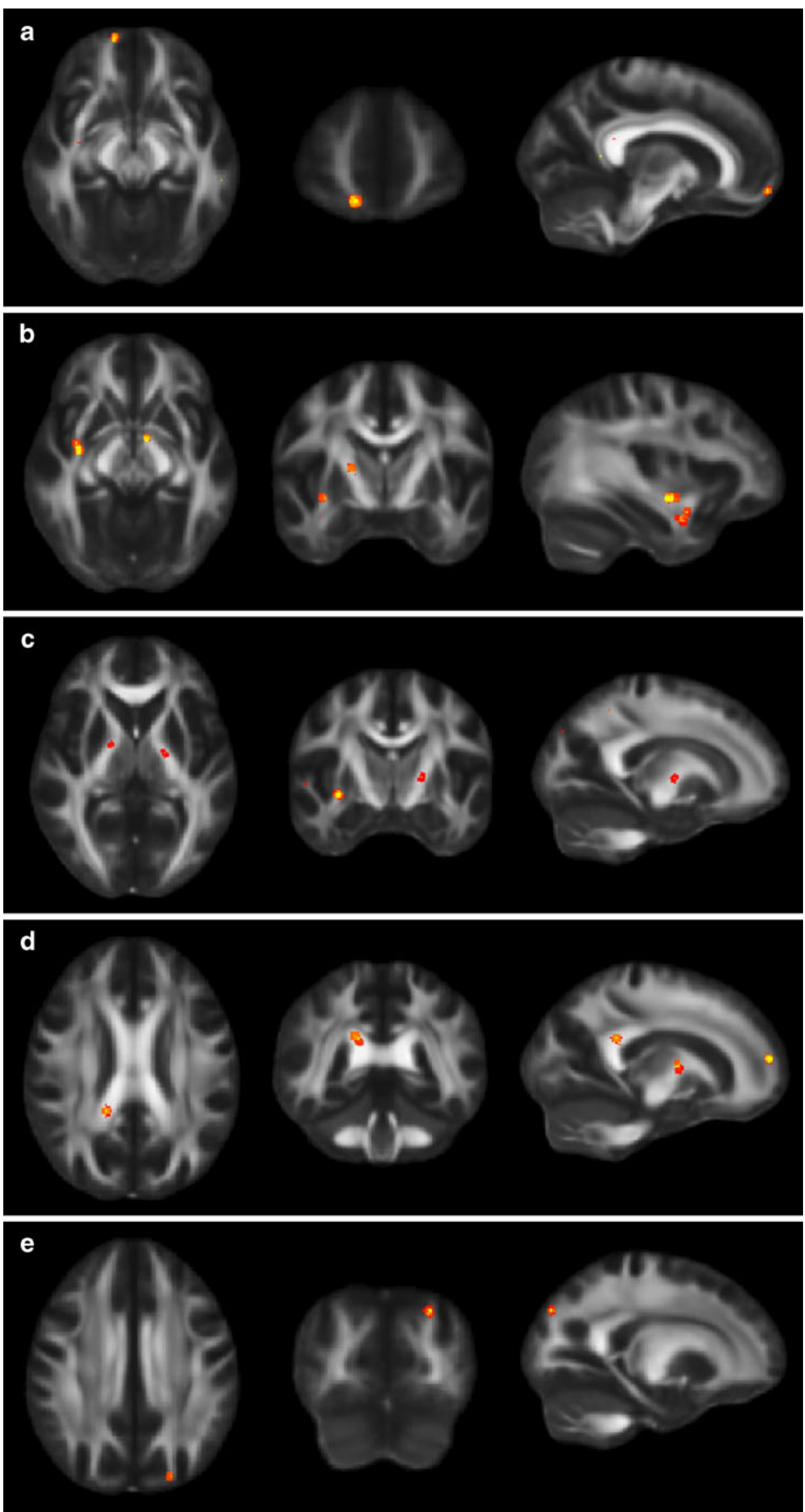




Fig. 4 Three-dimensional, high-resolution gradient recalled echo T2*weighted images from one patient showing two microhaemorrhages (arrows) in the right frontal lobe

patients was found to have microhaemorrhages, possibly indicating true shearing injury in this sole patient, it is not likely that our findings reflect true shearing injury leading to primary axotomy, but rather reflect secondary axotomy and pathological white matter structure due to stretching, straining or deformation forces.

We also found an increase of MD associated with the severity of post-concussive symptoms in the IFO, ILF and SLF. Previous reports of localised diffusivity alterations are inconsistent, presumably reflecting the heterogeneity of patient study groups and varying degrees of head injury studied. Both increases and decreases of diffusivity have been observed in patients with head injury [25, 31, 33, 58]. Also, the sensitivity of DWI for white matter injury after head injury has not been established. In the acute stage of minor head injury, Arfanakis et al. [31] reported an absence of significant alteration of diffusivity in relation to head injury, whereas others have reported changes in diffusivity to be a more sensitive measure than anisotropy changes for white matter injury detection $[33,59]$. Furthermore, DWI of known diffuse axonal injury lesions demonstrated concomitant reductions in diffusivity in only $65 \%$ of shearing injuries [25], suggesting a lack of sensitivity of this measure. Physiological mechanisms responsible for both decreases and increases in diffusivity have been proposed. Decreases have been attributed to trauma-induced changes in tissue metabolism as well as trauma-induced ischemia, in turn leading to cytotoxic oedema [58]. Trauma-induced axotomy with the formation of retraction balls and concomitant cytoskeletal collapse along severed axons may be another explanation for the reduction of diffusivity. Increases in diffusivity are thought to represent vasogenic oedema, possibly in relation with less severe injury, or to be due to increased molecular mobility as a result of neuronal or glial loss in the later stages of injury [30].
There are some reports correlating white matter changes with clinical measures after head injury. Poor performance on learning and memory indices were previously reported to be correlated with increased diffusivity in the posterior cingulate, the hippocampal formation and the temporal, frontal and occipital cortex in a study by Salmond et al. [60]. In patients who had recovered from coma, Nakayama et al. [61] found that reduction of anisotropy in the splenium of the corpus callosum correlated with the MMSE. Kraus et al. [34] found a modest negative correlation between FA and executive function, attention and memory, whilst $\mathrm{Wu}$ et al. [62] reported a weak correlation between ADC values in the cingulum bundle and memory function in acute minor head injury.

Recently, several studies similar to our study were published correlating DTI findings with post-concussive symptoms in moderate and mild head injury patients [59, 63-66]. In contrast to our study, however, patients with macrostructural findings were generally not excluded and imaging was performed at earlier stages post-injury. In the early stages after minor head injury, Chu et al. [64], Bazarian et al. [63], Mayer et al. [65] and Wilde et al. [66] found increases of FA and decreases of MD in relation to the severity of post-concussive symptoms in the corpus callosum, $[63,64,66]$, the posterior limb of the internal capsule [63], as well as several other white matter regions [66]. The fact that FA was increased, as opposed to the expected decrease with loss of white matter integrity, is likely due to the timing of imaging very early post-injury (1-10 days), when cytotoxic oedema and localised inflammatory responses prevail and white matter degradation has not had time to develop. This is further supported by findings of decreased radial diffusivity in areas of increased FA [65]. In the subacute phase (15 days post-injury), Messé et al. [59] only found MD increases and no FA changes in relation to the severity of post-concussive symptoms, again possibly reflecting the pathophysiological time course of the several stages of white matter injury. Our findings of increased diffusivity as well as decreased anisotropic diffusion in areas shown to be affected by diffuse axonal injury support the hypothesis that post-concussive symptoms are a manifestation of traumatic axonal injury, even in the absence of macrostructural evidence of brain injury from conventional neuroimaging techniques.

The correlation of microstructural white matter injury with neuropsychological measures of cognition in mildly $[62,67]$ and more severely injured patients $[34,60,61]$ provides further evidence that microstructural white matter injury results in cognitive deficits. Such neurocognitive deficits, if present following minor head injury, are generally subtle, most commonly affecting working memory and selective attention, which are essential for normal functioning in everyday life [68]. This is reflected in the time course of 
post-concussive complaints which, after an initial spontaneous decrease over the course of several weeks after the injury, typically aggravate when patients resume their normal, and more demanding, activities, such as return to work or school [6]. In our previous study of minor head injury patients using functional MRI to assess the neural correlate of working memory, patients with more severe post-concussive symptoms showed increased brain activity in the normal working memory network, as well as the recruitment of brain regions outside this network [68]. The findings are indicative of altered or multiple strategies used for working memory processing in order to counterbalance functional deficits in working memory processing as a result of-microstructural-injury. In the present study, only limited testing of cognitive function was performed using the MMSE, with which subtle neurocognitive deficits are not likely to be detected. Testing cognition in the present study served solely to assess potential heterogeneity, and thus confounding across subgroups, and not to evaluate neuropsychological deficits after MHI, studies of which are already numerous [6-8]. We feel that using MMSE and educational level as crude measures of cognitive function were sufficient for the purpose of this study.

To control for the heterogeneity of this patient population, conservative selection criteria were employed, resulting in the study's modest statistical power. First, highresolution T1-weighted and sensitive FLAIR imaging were used to exclude patients with any identifiable white matter abnormalities, thereby restricting the number of patients eligible for inclusion. Furthermore, only patients with (near-)optimal GCS scores were recruited, further biasing the sample away from obvious clinical manifestations of head injury and towards subtle symptomatology characteristic of the post-concussion syndrome. This is in contrast with previous studies which either lacked exclusion criteria for traumatic abnormalities or used less sensitive imaging sequences such as the anatomical T1-weighted image to exclude patients with traumatic abnormalities. Although the statistical power of our study may not be as great as would be desired, we did control for false positive errors by limiting ourselves to an assessment of the white matter tracts only by using TBSS and by applying a correction for multiple comparisons on a cluster level.

\section{Conclusion}

We found that the severity of post-concussive symptoms after minor head injury was significantly correlated with a reduction of white matter integrity, as manifested by increases in diffusivity and reduced anisotropic diffusion. Our findings provide evidence of microstructural injury as a neuropathological substrate of the post-concussion syndrome.
Acknowledgements The authors wish to thank the trial nurses of the department of Radiology, Mrs. W.J. van Leeuwen and Mrs. C. van Bavel-van Hamburg, for their contribution to participant recruitment.

Conflict of interest statement We declare that we have no conflict of interest.

Open Access This article is distributed under the terms of the Creative Commons Attribution Noncommercial License which permits any noncommercial use, distribution, and reproduction in any medium, provided the original author(s) and source are credited.

\section{References}

1. Cassidy J, Carroll L, Peloso P, Borg J, von Holst H et al (2004) Incidence, risk factors and prevention of mild traumatic brain injury: results of the WHO Collaborating Centre Task Force on Mild Traumatic Brain Injury. J Rehabil Med (43 Suppl):28-60 (Feb)

2. Alexander MP (1995) Mild traumatic brain injury: pathophysiology, natural history, and clinical management. Neurology 45:1253-1260

3. Alexander MP (1997) Minor traumatic brain injury: a review of physiogenesis and psychogenesis. Semin Clin Neuropsychiatry 2:177-187

4. Dikmen S, McLean A, Temkin N (1986) Neuropsychological and psychosocial consequences of minor head injury. J Neurol Neurosurg Psychiatry 49:1227-1232

5. WHO (1993) The ICD-10 classification of mental and behavioural disorders. Diagnostic criteria for research

6. Bohnen N, Jolles J, Twijnstra A (1992) Neuropsychological deficits in patients with persistent symptoms six months after mild head injury. Neurosurgery 30:692-696

7. Cicerone KD, Azulay J (2002) Diagnostic utility of attention measures in postconcussion syndrome. Clin Neuropsychol $16: 280-289$

8. Bohnen N, Twijnstra A, Jolles J (1992) Performance in the Stroop color word test in relationship to the persistence of symptoms following mild head injury. Acta Neurol Scand 85:116-121

9. Hofman PAM, Stapert SZ, MJPGv K, Jolles J, Jd K et al (2001) MR imaging, single-photon emission $\mathrm{CT}$, and neurocognitive performance after mild traumatic brain injury. Am J Neuroradiol 22:441-449

10. Bohnen N, Jolles J (1992) Neurobehavioral aspects of postconcussive symptoms after mild head injury. J Nerv Ment Dis 180:683-692

11. Bazarian JJ, Wong T, Harris M, Leahey N, Mookerjee S et al (1999) Epidemiology and predictors of post-concussive syndrome after minor head injury in an emergency population. Brain Inj 13:173-189

12. Bigler ED (2001) Distinguished neuropsychologist award lecture 1999. The lesion(s) in traumatic brain injury: implications for clinical neuropsychology. Arch Clin Neuropsychol 16:95-131

13. Bigler ED (2003) Neurobiology and neuropathology underlie the neuropsychological deficits associated with traumatic brain injury. Arch Clin Neuropsychol 18:595-621, discussion 623-627

14. Lishman WA (1988) Physiogenesis and psychogenesis in the 'post-concussional syndrome'. Br J Psychiatry 153:460-469

15. King N (1997) Mild head injury: neuropathology, sequelae, measurement and recovery. Br J Clin Psychol 36(Pt 2):161-184

16. Szymanski HV, Linn R (1992) A review of the postconcussion syndrome. Int J Psychiatry Med 22:357-375

17. Metting Z, Rodiger LA, Stewart RE, Oudkerk M, De Keyser J et al (2009) Perfusion computed tomography in the acute phase of mild head injury: regional dysfunction and prognostic value. Ann Neurol 66:809-816 
18. Hofman PAM, Verhey FRJ, Wilmink JT, Rozendaal N, Jolles J (2002) Brain lesions in patients visiting a memory clinic with postconcussional sequelae after mild to moderate brain injury. $\mathrm{J}$ Neuropsychiatry Clin Neurosci 14:176-184

19. Heitger MH, Jones RD, Macleod AD, Snell DL, Frampton CM et al (2009) Impaired eye movements in post-concussion syndrome indicate suboptimal brain function beyond the influence of depression, malingering or intellectual ability. Brain 132:2850-2870

20. Pontifex MB, O'Connor PM, Broglio SP, Hillman CH (2009) The association between mild traumatic brain injury history and cognitive control. Neuropsychologia 47:3210-3216

21. Savola O, Hillbom M (2002) Early predictors of post-concussion symptoms in patients with mild head injury. Eur J Neurol 10:175181

22. Kruijk JRd, Leffers P, Menheere PPCA, Meerhoff S, Rutten J et al (2002) Prediction of post-traumatic complaints after mild traumatic brain injury: early symptoms and biochemical markers. J Neurol Neurosurg Psychiatry 73:727-732

23. Lipton ML, Gulko E, Zimmerman ME, Friedman BW, Kim M et al (2009) Diffusion-tensor imaging implicates prefrontal axonal injury in executive function impairment following very mild traumatic brain injury. Radiology 252:816-824

24. Kinoshita T, Moritani T, Hiwatashi A, Wang HZ, Shrier DA et al (2005) Conspicuity of diffuse axonal injury lesions on diffusionweighted MR imaging. Eur J Radiol 56:5-11

25. Huisman TAGM, Sorensen AG, Hergan K, Gonzalez RG, Schaefer PW (2003) Diffusion-weighted imaging for the evaluation of diffuse axonal injury in closed head injury. J Comput Assist Tomogr 27:5-11

26. Lipton ML, Gellella E, Lo C, Gold T, Ardekani BA et al (2008) Multifocal white matter ultrastructural abnormalities in mild traumatic brain injury with cognitive disability: a voxel-wise analysis of diffusion tensor imaging. J Neurotrauma 25:1335-1342

27. Lee H, Wintermark M, Gean AD, Ghajar J, Manley GT et al (2008) Focal lesions in acute mild traumatic brain injury and neurocognitive outcome: CT versus 3 T MRI. J Neurotrauma 25:1049-1056

28. Rauscher A, Sedlacik J, Barth M, Haacke EM, Reichenbach JR (2005) Nonnvasive assessment of vascular architecture and function during modulated blood oxygenation using susceptibility weighted magnetic resonance imaging. Magn Reson Med 54:87-95

29. Reichenbach JR, Venkatesan R, Schillinger DJ, Kido DK, Haacke EM (1997) Small vessels in the human brain: MR venography with deoxyhemoglobin as an intrinsic contrast agent. Radiology 204:272-277

30. Chan JHM, Tsui EYK, Peh WCG, Fong D, Fok KF et al (2003) Diffuse axonal injury: detection of changes in anisotropy of water diffusion by diffusion-weighted imaging. Neuroradiology 45:34-38

31. Arfanakis K, Haughton VM, Carew JD, Rogers BP, Dempsey RJ et al (2002) Diffusion tensor MR imaging in diffuse axonal injury. Am J Neuroradiol 23:794-802

32. Chappell MH, Ulug AM, Zhang L, Heitger MH, Jordan BD et al (2006) Distribution of microstructural damage in the brains of professional boxers: a diffusion MRI study. J Magn Reson Imaging 24:537-542

33. Xu J, Rasmussen IA, Lagopoulos J, Haberg A (2007) Diffuse axonal injury in severe traumatic brain injury visualized using high-resolution diffusion tensor imaging. J Neurotrauma 24:753-765

34. Kraus MF, Susmaras T, Caughlin BP, Walker CJ, Sweeney JA et al (2007) White matter integrity and cognition in chronic traumatic brain injury: a diffusion tensor imaging study. Brain 130:2508-2519

35. Huisman TA (2003) Diffusion-weighted imaging: basic concepts and application in cerebral stroke and head trauma. Eur Radiol 13:2283-2297
36. Basser PJ, Jones DK (2002) Diffusion-tensor MRI: theory, experimental design and data analysis - a technical review. NMR Biomed 15:456-467

37. Pierpaoli C, Jezzard P, Basser PJ, Barnett A, Di Chiro G (1996) Diffusion tensor MR imaging of the human brain. Radiology 201:637-648

38. Benson RR, Meda SA, Vasudevan S, Kou Z, Govindarajan KA et al (2007) Global white matter analysis of diffusion tensor images is predictive of injury severity in traumatic brain injury. J Neurotrauma 24:446-459

39. Tong KA, Ashwal S, Holshouser BA, Shutter LA, Herigault G et al (2003) Hemorrhagic shearing lesions in children and adolescents with posttraumatic diffuse axonal injury: improved detection and initial results. Radiology 227:332-339

40. Vernooij M, van der Lugt A, Ikram M, Niessen W, Hofman A et al (2008) Prevalence and risk factors of cerebral microbleeds: the Rotterdam Scan Study. Neurology 70:1208-1214

41. Vernooij M, Ikram M, Wielopolski P, Krestin G, Breteler M et al (2008) Cerebral microbleeds: accelerated 3D T2*-weighted GRE MR imaging versus conventional 2D T2*-weighted GRE MR imaging for detection. Radiology 248:272-277

42. Folstein MF, Folstein SE, McHugh PR (1975) Mini-mental state. A practical method for grading the cognitive state of patients for the clinician. J Psychiatr Res 12:189-198

43. King NS, Crawford S, Wenden FJ, Moss NEG, Wade DT (1995) The Rivermead Post concussion Symptoms Questionnaire: a measure of symptoms commonly experienced after head injury and its reliability. J Neurol 242:587-592

44. Ingebrigtsen T, Waterloo K, Marup-Jendsen S, Attner E, Romner B (1998) Quantification of post-concussion symptoms 3 months after minor head injury in 100 consecutive patients. J Neurol 245:609-612

45. D'Esposito M, Detre JA, Alsop DC, Shin RK, Atlas S et al (1995) The neural basis of the central executive system of working memory. Nature 378:279-281

46. Parizel PM, Ozsarlak, Van Goethoem JW, van den Hauwe L, Dillen $C$ et al (1998) Imaging findings in diffuse axonal injury after closed head trauma. Eur Radiol 8:960-965

47. Smith SM, Jenkinson M, Johansen-Berg H, Rueckert D, Nichols TE et al (2006) Tract-based spatial statistics: voxelwise analysis of multi-subject diffusion data. Neuroimage 31:1487-1505

48. Smith SM, Jenkinson M, Woolrich MW, Beckmann CF, Behrens TE et al (2004) Advances in functional and structural MR image analysis and implementation as FSL. Neuroimage 23(Suppl 1): S208-S219

49. Smith SM (2002) Fast robust automated brain extraction. Hum Brain Mapp 17:143-155

50. Rueckert D, Sonoda LI, Hayes C, Hill DL, Leach MO et al (1999) Nonrigid registration using free-form deformations: application to breast MR images. IEEE Trans Med Imaging 18:712-721

51. Rueckert D (2007) Image registration toolkit. www.doc.ic.ac.uk/ dr/ software, accessed 6 January 2008

52. Hermoye L, Laurent J-P, Cosnard G, Wakana S, Jiang $\mathrm{H}$ et al. (2004). DTI atlas. www.dtiatlas.org, accessed 6 January 2008

53. Wakana S, Jiang H, Nagae-Poetscher LM, van Zijl PC, Mori S (2004) Fiber tract-based atlas of human white matter anatomy. Radiology 230:77-87

54. Mac Donald CL, Dikranian K, Song SK, Bayly PV, Holtzman DM et al (2007) Detection of traumatic axonal injury with diffusion tensor imaging in a mouse model of traumatic brain injury. Exp Neurol 205:116-131

55. Lo C, Shifteh K, Gold T, Bello JA, Lipton ML (2009) Diffusion tensor imaging abnormalities in patients with mild traumatic brain injury and neurocognitive impairment. J Comput Assist Tomogr $33: 293-297$ 
56. Niogi SN, Mukherjee P, Ghajar J, Johnson C, Kolster RA et al (2008) Extent of microstructural white matter injury in postconcussive syndrome correlates with impaired cognitive reaction time: a $3 \mathrm{~T}$ diffusion tensor imaging study of mild traumatic brain injury. AJNR Am J Neuroradiol 29:967-973

57. Inglese M, Makani S, Johnson G, Cohen BA, Silver JA et al (2005) Diffuse axonal injury in mild traumatic brain injury: a diffusion tensor imaging study. J Neurosurg 103:298-303

58. Ito J, Marmarou A, Barzo P, Fatouros P, Corwin F (1996) Characterization of edema by diffusion-weighted imaging in experimental traumatic brain injury. J Neurosurg 84:97-103

59. Messé A, Caplain S, Paradot G, Garrigue D, Mineo J-F et al. (2010) Diffusion tensor imaging and white matter lesions at the subacute stage in mild traumatic brain injury with persistent neurobehavioral impairment. Hum Brain Mapp. doi:10.1002/hbm.21092

60. Salmond CH, Menon DK, Chatfield DA, Williams GB, Pena A et al (2006) Diffusion tensor imaging in chronic head injury survivors: correlations with learning and memory indices. Neuroimage 29:117-124

61. Nakayama N, Okumura A, Shinoda J, Yasokawa YT, Miwa K et al (2006) Evidence for white matter disruption in traumatic brain injury without macroscopic lesions. J Neurol Neurosurg Psychiatry $77: 850-855$
62. Wu TC, Wilde EA, Bigler ED, Yallampalli R, McCauley SR et al (2010) Evaluating the relationship between memory functioning and cingulum bundles in acute mild traumatic brain injury using diffusion tensor imaging. J Neurotrauma 27:303-307

63. Bazarian JJ, Zhong J, Blyth B, Zhu T, Kavcic V et al (2007) Diffusion tensor imaging detects clinically important axonal damage after mild traumatic brain injury: a pilot study. J Neurotrauma 24:1447-1459

64. Chu Z, Wilde EA, Hunter JV, McCauley SR, Bigler ED et al (2010) Voxel-based analysis of diffusion tensor imaging in mild traumatic brain injury in adolescents. AJNR Am J Neuroradiol 31:340-346

65. Mayer AR, Ling J, Mannell MV, Gasparovic C, Phillips JP et al (2010) A prospective diffusion tensor imaging study in mild traumatic brain injury. Neurology 74:643-650

66. Wilde EA, McCauley SR, Hunter JV, Bigler ED, Chu Z et al (2008) Diffusion tensor imaging of acute mild traumatic brain injury in adolescents. Neurology 70:948-955

67. Miles L, Grossman RI, Johnson G, Babb JS, Diller L et al (2008) Short-term DTI predictors of cognitive dysfunction in mild traumatic brain injury. Brain Inj 22:115-122

68. Smits M, Dippel DWJ, Houston GC, Wielopolski PA, Koudstaal PJ et al (2009) Postconcussion syndrome after minor head injury: brain activation of working memory and attention. Hum Brain Mapp 30:2789-2803 